\title{
Seminal Ideas in Integral Methods
}

\author{
G.M.L. Gladwell
}

\section{Introduction}

When we write papers we claim that we are the author, even sole author, of the analysis we present. In this paper, I intend to show how farcical this claim is; every step in our analysis is usually the end result of work painstakingly done by our antecedents over the last $21 / 2$ millennia. At the end of the paper I will identify the mathematicians responsible for the crucial steps in the analysis of a paper I wrote recently. The historical material is largely taken from Kline [1].

Integral methods have their genesis in the work of Eudoxus (408-335 B.C.). By inscribing and circumscribing polygons, he obtained lower and upper bounds of areas bounded by circles, and introduced the method of exhaustion. The polymath Archimedes of Syracuse (287-212 B.C.) developed this method, applying it to volumes and areas, centres of gravity, flotation, pumps, astronomy, etc. He is justly placed among the three great mathematicians of all time.

Skipping almost 2 millenia, we arrrive at the 17th Century explosion of knowledge. After the forerunners Pierre Fermat (1601-1665), John Wallis (1616-1703), Blaise Pascal (1623-1662) and Isaac Barrow (16301677) came the luminaries Isaac Newton (1642-1727) and Gottfried Leibniz (1646-1716). For two hundred years England and France were at loggerheads over who, Newton or Leibniz, was the inventor of the differential and integral calculus as we know it. The answer is undoubtedly, both. Both independently recognized that differentiation and integration were inverses of each other. While the intuitive-practical Newton buttressed the results he obtained from his calculus with the geometrical methods of Archimedes, the more philosophical Leibniz obtained the various rules, e.g., for integration by parts, and considered maxima and minima. Newton's greatness lay in his combining the calculus with his newly found Laws of Motion and the Inverse Square Law of Gravitation. 


\section{Fourier (?) Transforms}

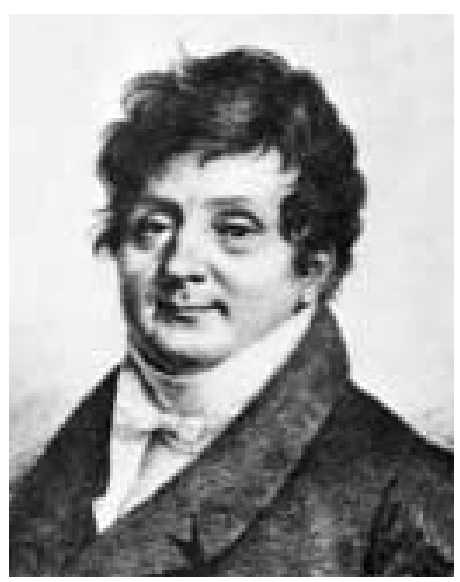

Joseph Fourier (1768-1830)

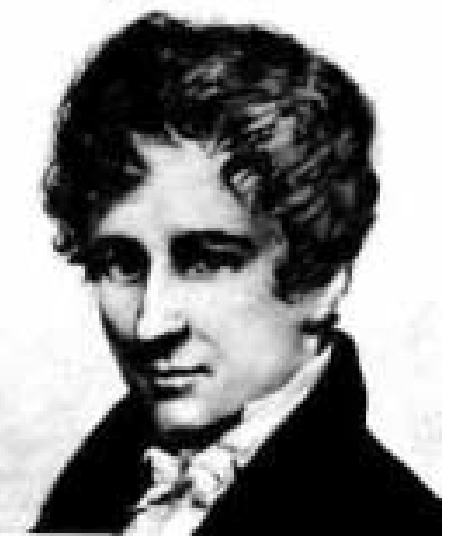

Niels Abel (1802-1829)

There is a Fundamental Theorem of the History of Mathematics:

$A$ 's Theorem is due to. $B$, where $B \neq A$.

It has a Corollary:

The $A, B$ Theorem is due to. $C$, where $C \neq A, C \neq B$.

A remarkable illustration of this 'Theorem' is provided by the results attributed to Joseph Fourier (1768-1830). Today Fourier's name is firmly attached to Fourier Series and the Fourier Integral. However, as early as 1729, 39 years before Fourier's birth, Leonhard Euler (1707-1783) had used the series representation

$$
f(x)=\frac{1}{2} a_{0}+\sum_{n=1}^{\infty}\left(a_{n} \cos n x+b_{n} \sin n x\right)
$$

for interpolation, and had obtained the formulae

$$
a_{n}=\frac{1}{\pi} \int_{0}^{2 \pi} f(x) \cos n x d x, \quad b_{n}=\frac{1}{\pi} \int_{0}^{2 \pi} f(x) \sin n x d x,
$$

albeit by complicated infinite series arguments; he had used orthogonality to obtain $a_{n}, b_{n}$ by 1777 .

Fourier's essential contribution (1811) was his use of the trigonometric series

$$
T(x, t)=\sum_{n=1}^{\infty} b_{n} \exp \left[-\left(n^{2} \pi^{2} / k^{2} L^{2}\right) t\right] \sin (n \pi x / L)
$$


to solve the heat equation

$$
\frac{\partial^{2} T}{\partial x^{2}}=k^{2} \frac{\partial T}{\partial t}, \quad T(0, t)=0=T(L, t)
$$

The initial condition $T(x, 0)=f(x)$ gives

$$
f(x)=\sum_{n=1}^{\infty} b_{n} \sin (n \pi x / L)
$$

with the inversion formula

$$
b_{n}=\frac{2}{L} \int_{0}^{L} f(x) \sin (n \pi s / L) d s
$$

Fourier made a heuristic jump by supposing $L \rightarrow \infty$; the series become an integral; introducing a normalizing factor $\left(\frac{2}{\pi}\right)^{\frac{1}{2}}$ we write

$$
T(x, t)=\left(\frac{2}{\pi}\right)^{\frac{1}{2}} \int_{0}^{\infty} Q(q) \exp \left(-k^{2} q^{2} t\right) \sin q x d q
$$

which in today's notation we write as

$$
T(x, t)=\mathcal{F}_{s}\left[Q(q) \exp \left(-k^{2} q^{2} t\right) ; q \rightarrow x\right]
$$

we have the transform pair

$$
f(x)=\mathcal{F}_{s}[Q(q) ; q \rightarrow x], Q(q)=\mathcal{F}_{s}[f(x) ; x \rightarrow q]
$$

showing that $\mathcal{F}_{s}$ (and similarly $\mathcal{F}_{c}$ ) are their own inverses:

$$
\mathcal{F}_{x}^{-1}=\mathcal{F}_{s}, \quad \mathcal{F}_{c}^{-1}=\mathcal{F}_{c}
$$

The engineer cum mathematician Fourier valued results more than rigour. This rigour was found only after attention was directed to the complex plane, as we show in Section 3 .

Neils Henrig Abel (1802-1829) is an outlier in the development of integral methods. He found the time of descent $T(y)$ of a particle falling freely down a curve with equation $s=f(\eta)$, as shown in Fig. 1, from 
$P(x, y)$ to 0 .

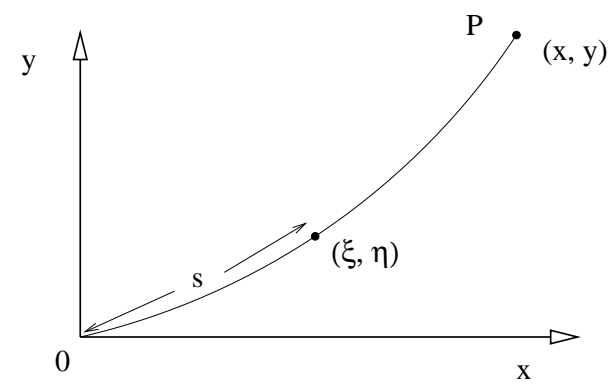

Fig. 1. A particle falls from $P(x, y)$ to 0 in time $T(y)$.

Conservation of energy gives

$$
F(y)=\sqrt{2 g} T(y)=\int_{0}^{y} \frac{f^{\prime}(\eta) d \eta}{(y-\eta)^{\frac{1}{2}}}
$$

Abel inverted this integral equation:

$$
f(\eta)=\frac{1}{\pi} \int_{0}^{\eta} \frac{F(\eta) d \eta}{(\eta-y)^{\frac{1}{2}}}
$$

This constructs the curve from the descent time; we will return to this pair of equations later.

\section{Complex Integrals}

Carl Friedrick Gauss (1777-1855) is the third of the great three: Archimedes, Newton and Gauss. It was he who finally answered the question What is a number? His proof of the fundamental theorem of algebra, and his elucidation of the role of complex numbers was the culmination of a generation of work due to Alexandre-Theophile Vandermonde (17351796), Jean-Robert Argand (1768-1822) as well as Poisson and Cauchy. The emergence of complex numbers led to the concepts of complex functions and complex (contour) integrals. It is impossible to describe the tortuous journey of discovery of such matters in these few pages; it occupied the greatest minds of the 19th Century and was completed only with the work of Karl Weierstrass (1815-1897) and Bernhard Riemann (1826-1866).

Cauchy showed that if $w$ is a function of $z=x+i y$, and

$$
w(z)=u(x, y)+i v(x, y)
$$

then the continuity of $u, v$ and their derivatives leads to what we now call 
the Cauchy-Riemann equations

$$
\frac{\partial u}{\partial x}=\frac{\partial v}{\partial y}, \quad \frac{\partial u}{\partial y}=-\frac{\partial v}{\partial x}
$$

showing that both $u(x, y)$ and $v(x, y)$ satisfy Laplace's equation

$$
\frac{\partial^{2} u}{\partial x^{2}}+\frac{\partial^{2} u}{\partial y^{2}}=0
$$

Starting from

$$
w(z)=\exp (i z)=\exp (i x) \exp (-y)=(\cos x+i \sin x) \exp (-y)
$$

it is a simple step to obtain the solution

$$
u(x, y)=\mathcal{F}_{c}[\exp (-\xi y) F(\xi) ; \xi \rightarrow x]
$$

If $u(x, y)$ satisfies the boundary condition $u(x, 0)=f(x)$ then

$$
\mathcal{F}_{c}[F(\xi) ; \xi \rightarrow x]=f(x)
$$

which has the solution

$$
F(\xi)=\mathcal{F}_{c}[f(x) ; x \rightarrow \xi]
$$

It seems to have been Poisson who first studied complex integrals, and Gauss and Cauchy who showed that if $f(z)$ is holomorphic and $c$ is a closed path then,

$$
\int_{c} f(z) d z=0 .
$$

Cauchy may or may not be responsible for his residue theorem, that if

$$
f(z)=\frac{a}{z-z_{0}}+\text { holomorphic }
$$

then

$$
\frac{1}{2 \pi i} \int_{c} f(z) d z=a
$$

for a path $c$ around $z_{0}$. Curiously, the concept of a complex integral led to the evaluation of many real integrals, and to the proper definitions of logarithms of negative and complex numbers. Today many special functions are defined as complex integrals. 


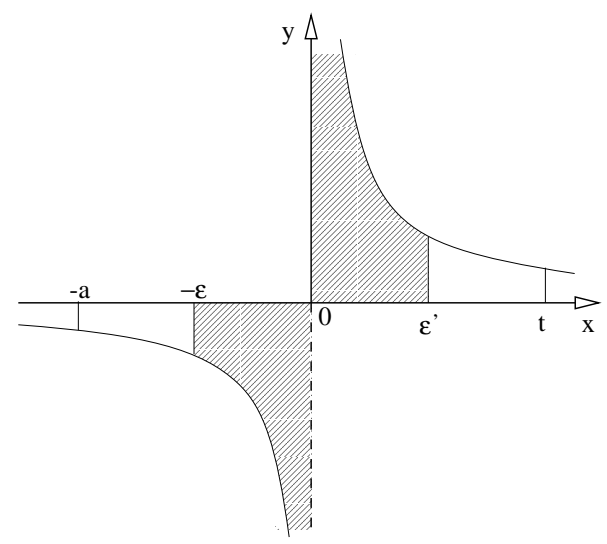

Fig. 2 When $\varepsilon=\varepsilon^{\prime}$, the two shaded areas are equal.

In this short note we cannot discuss the evolution of the definition of integral arising from the work of Riemann or Henri Lebesque (18751941). Instead, for our particular concern, we note the definition of the Cauchy Principal Value (PV) of a real function with a simple pole at the origin. Figure 2 shows the graph of $\frac{1}{x}$. We have

$$
\begin{aligned}
\int_{-a}^{b} \frac{d x}{x} & =\lim _{\varepsilon, \varepsilon^{\prime} \rightarrow 0}\left\{\int_{-a}^{-\varepsilon} \frac{d x}{x}+\int_{\varepsilon^{\prime}}^{b} \frac{d x}{x}\right\} \\
& =\lim _{\varepsilon, \varepsilon^{\prime} \rightarrow 0}\left\{\ln \left(\frac{\varepsilon}{a}\right)+\ln \left(\frac{b}{\varepsilon^{\prime}}\right)\right. \\
& =\lim _{\varepsilon, \varepsilon^{\prime} \rightarrow 0}\left\{\ln \left(\frac{b}{a}\right)+\ln \left(\frac{\varepsilon}{\varepsilon^{\prime}}\right)\right\} .
\end{aligned}
$$

The limit does not exist, but if $\varepsilon^{\prime}=\varepsilon$, then it does; we define the principal value of the integral as that limit, and then

$$
\text { P.V. } \int_{-a}^{b} \frac{d x}{x}=\ln \left(\frac{b}{a}\right) .
$$

The Principal Value comes into its own in the problem of finding the limits of the complex integral

$$
F(z)=\frac{1}{2 \pi i} \int_{c} \frac{f(t) d t}{t-z}
$$

as $z$ approaches the contour $C$ from $S^{+}$or $S^{-}$, as shown in Figure 3. J. Plemelj [2] first gave the equations

$$
F^{+}\left(t_{0}\right)+F^{-}\left(t_{0}\right)=\frac{1}{\pi} \int_{C} \frac{f(t) d t}{t-t_{0}}
$$




$$
F^{+}\left(t_{0}\right)-F^{-}\left(t_{0}\right)=f\left(t_{0}\right)
$$

The integral in (6) must be interpreted as a P.V.

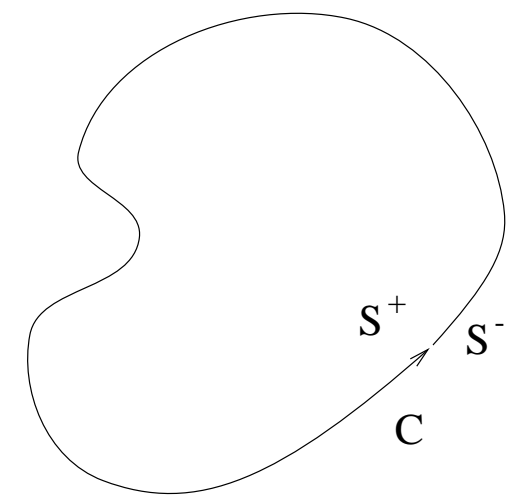

Fig. $3 z$ approaches $C$ from $S^{+}$or $S^{-}$.

The problem of finding a function $\Phi(z)$ which is holomorphic in the plane cut along an arc or contour $L$, and which has a specified linear relationship

$$
\kappa \Phi^{+}(t)+\Phi^{-}(t)=f(t), \quad t \varepsilon L,
$$

between its boundary values on either side of $L$, is attributed variously to Riemann or David Hilbert (1862-1943); its solution was due largely to the work of N.I. Muskhelishvili [3]. In the simple case in which $L=(-1,1)$, we have

$$
\begin{gathered}
\kappa \Phi^{+}(t)+\Phi^{-}(t)=f(t), \quad t \varepsilon L \\
\Phi^{+}(t)-\Phi^{-}(t)=0, \quad t \varepsilon L^{\prime}
\end{gathered}
$$

Muskhelishvili introduced the function

$$
X(z)=(z-1)^{-\frac{1}{2}+i \alpha}(z+1)^{-\frac{1}{2}-i \alpha},
$$

which has branch points \pm 1 , and which satisfies

$$
\begin{array}{cc}
\kappa X^{+}(t)+X^{-}(t)=0 & t \varepsilon L, \\
X^{+}(t)-X^{-}(t)=0 & t \varepsilon L^{\prime},
\end{array}
$$

where $\kappa=\exp (2 \pi \alpha)$, to obtain the equation

$$
\Omega^{+}(t)-\Omega^{-}(t)= \begin{cases}f(t) /\left(\kappa X^{+}(t)\right) & \text { on } L \\ 0, & \text { on } L^{\prime}\end{cases}
$$

for the quotient $\Omega(z)=\Phi(z) / X(z)$. Now the Plemelj formula (6) gives

$$
\Omega(z)=\frac{1}{2 \pi i \kappa} \int_{L} \frac{f(t) d t}{X^{+}(t)(t-z)}+P_{n}(z)
$$


where $P_{n}(z)$ is a polynomial.

\section{Return to Transforms}

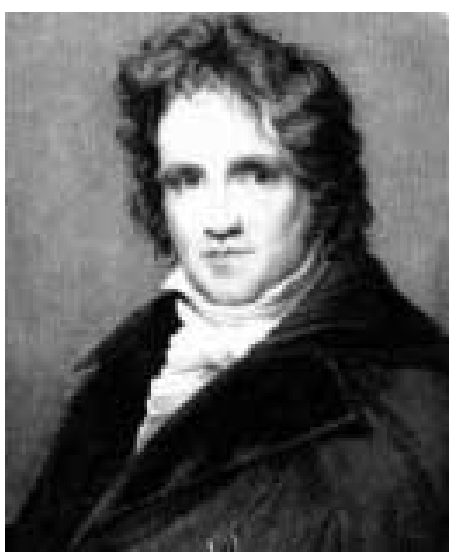

Wilhelm Bessel (1784-1846)

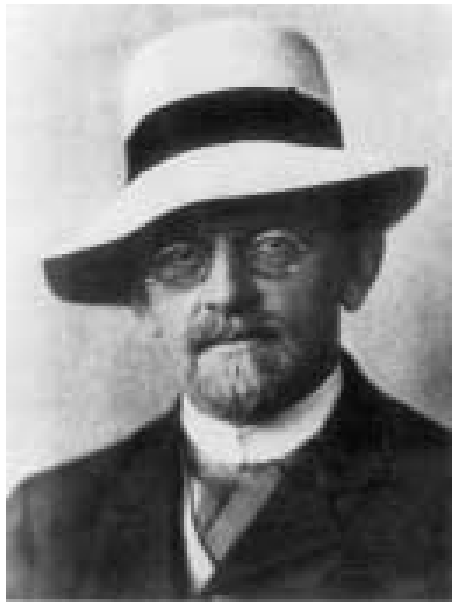

David Hilbert (1862-1943)

While Cauchy had studied the 2D Laplace's equation (3), the mathematical astronomer Wilhelm Bessel (1784-1846) studied the 3D version

$$
\frac{\partial^{2} u}{\partial x^{2}}+\frac{\partial^{2} u}{\partial y^{2}}+\frac{\partial^{2} u}{\partial z^{2}}=0
$$

The equation is separable in cylindrical polar coordinates $(r, \theta, z)$ and has solution

$$
u(r, \theta, z)=\mathcal{H}_{n}\left\{\begin{array}{c}
\cos n \theta \\
\sin n \theta
\end{array} \quad \exp ( \pm \xi z) f(\xi) ; \xi \rightarrow r\right\}
$$

where

$$
\mathcal{H}_{n}\{f(\xi) ; \xi \rightarrow r\}=\int_{0}^{\infty} \xi f(\xi) J_{n}(\xi r) d \xi,
$$

and $J_{n}(\xi r)$ is a solution of

$$
r^{2} R^{\prime \prime}+r R^{\prime}+\left(\xi^{2} r^{2}-n^{2}\right) R=0 .
$$

For integer values of $n, J_{n}(x)$ is related to sines and cosines through

$$
J_{n}(x)=\frac{1}{2 \pi} \int_{0}^{2 \pi} \cos (n u-\sin u) d u,
$$


but, more importantly, the Hankel transform $\mathcal{H}_{n}$ named for Hermann Hankel (1839-1873), is its own inverse: $\mathcal{H}_{n}^{-1}=\mathcal{H}_{n}$.

We now have Fourier cosine, Fourier sine and Hankel transforms. How are they related?

First consider $\mathcal{F}_{c}$ and $\mathcal{F}_{s}$. Suppose

$$
\mathcal{F}_{s}[F(\xi) ; x]=f(x) H(a-x), \quad x \geq 0
$$

where $H(x)$ is the Heaviside function

$$
H(x)= \begin{cases}1, & x \geq 0 \\ 0, & x<0\end{cases}
$$

Extend $f(x)$ to $(-a, 0)$ as an odd function, then

$$
\mathcal{F}_{c}[F(\xi) ; x]=\frac{1}{\pi} \int_{-a}^{a} \frac{f(t) d t}{t-x}=h[f(t) ; x]
$$

The transform $h$ is called the Hilbert transform; it involves the real version of (4). Similarly, if

$$
\mathcal{F}_{c}[G(\xi) ; x]=g(x) H(a-x), x \leq 0,
$$

and $g(x)$ is extended to $(-a, 0)$ as an even function, then

$$
\mathcal{F}_{s}[G(\xi) ; x]=-h[g(t) ; x]
$$

To find out how the Fourier and Hankel transforms are related we must return to Abel! We define two variants of (1):

$$
\begin{aligned}
& \mathcal{A}_{1}[f(t) ; x]=\left(\frac{2}{\pi}\right)^{\frac{1}{2}} \int_{0}^{x}\left(x^{2}-t^{2}\right)^{-\frac{1}{2}} f(t) d t \\
& \mathcal{A}_{2}[f(t) ; x]=\left(\frac{2}{\pi}\right)^{\frac{1}{2}} \int_{0}^{\infty}\left(t^{2}-x^{2}\right)^{-\frac{1}{2}} f(t) d t .
\end{aligned}
$$

As in (1), (2), the inverses of $\mathcal{A}_{1}, \mathcal{A}_{2}$, which are examples of fractional integration operators (Sneddon [4]), involve derivatives of $\mathcal{A}_{1}, \mathcal{A}_{2}$ :

$$
\begin{gathered}
\mathcal{A}_{1}^{-1}[f(x) ; t]=D_{t} \mathcal{A}_{1}[x f(x) ; t], \\
\mathcal{A}_{2}^{-1}[f(x) ; t]=-D_{t} \mathcal{A}_{2}[x f(x) ; t],
\end{gathered}
$$

where $D_{t}=d / d t$. Now we have the remarkable results linking $\mathcal{F}_{c}, \mathcal{F}_{s}$ to the lowest Hankel transform $\mathcal{H}_{0}$ :

$$
\begin{aligned}
& \mathcal{H}_{0}\left[\xi^{-1} \mathcal{F}_{c}[f(t) ; \xi] ; x\right]=\mathcal{A}_{1}[f(t) ; x] \\
& \mathcal{H}_{0}\left[\xi^{-1} \mathcal{F}_{s}[f(t) ; \xi] ; x\right]=\mathcal{A}_{2}[f(t) ; x]
\end{aligned}
$$


Other relations, for $\mathcal{H}_{0}, \mathcal{H}_{1}, \mathcal{H}_{2}$, may be found in Tables 5.7.1 and 5.7.2 of Gladwell [5]. In operator notation, each of (9), (10), has the form $a b=c$; this immediately give 5 other relations:

$$
a=c b^{-1}, b=a^{-1} c, c^{-1}=b^{-1} a^{-1}, a^{-1}=b c^{-1}, b^{-1}=c^{-1} a
$$

We have linked Fourier, Bessel (Hankel), Hilbert and Abel!

In the 20th Century this analysis was placed on a secure foundation by Edward Titchmarsh (1899-1963). His student Ida Busbridge provided a crucial solution to dual integral equations. Ian Sneddon (1919-) simplified and extended the analysis of Titchmarsh and Busbridge, and applied it to potential problems in elasticity, thermodynames, etc. in his influential Fourier Transforms [6] and his little red book [4]. The compilation of tables of integral transforms owes much to the work of Harry Bateman (1882-1946) and Arthur Erdelyi (1908-1977).

\section{A case study}

We studied the problem of a penny-shaped rigid inclusion bonded to the interface between dissimilar isotropic half-spaces, as an example of the use of integral methods [7].

The problem is shown schematically in Figure 3 . The force $P$ pulls the inclusion up by an amount $h$; we need the force/displacement ratio $P / h$.

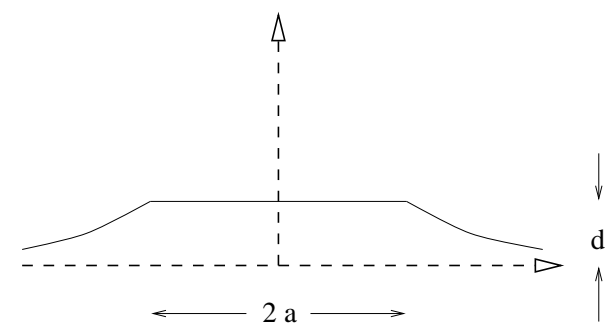

Fig. 4 The inclusion is pulled by a force $P$.

The reduction of the equations of elasticity to a single vector equation

$$
\operatorname{grad} \operatorname{div} \mathbf{D}+(1-2 \nu) \nabla^{2} \mathbf{D}=\mathbf{0}
$$

for the elastic displacement vector $\mathbf{D}$, is usually attributed to Claude Navier (1785-1836). The general solution

$$
2 \mu \mathbf{D}=4(1-\nu) \mathbf{q}-\nabla[(\mathbf{r} \cdot \mathbf{q})+\phi]
$$

in terms of two harmonic potentials, $\mathbf{q}$ and $\phi$, is due to P.F. Papkovich (1932) and H. Neuber (1934). 
The problem is axisymmetric; in cylindrical polar coordinates $r, \theta, z$, all quantities are independent of $\theta$. We write $[f] \equiv f(r, z=0+)-f(r, z=0-)$; in the usual notation, the boundary conditions on $z=0$ are

$$
\begin{gathered}
{[u]=0=[w],} \\
u=0, w=d, r<a ;\left[\tau_{r z}\right]=0=\left[\tau_{z z}\right], r>a .
\end{gathered}
$$

The Hankel transform solution of Laplace's equation leads to the integral equations

$$
\begin{aligned}
\mathcal{H}_{1}\left[\xi^{-1}\left(b_{1} L+b_{2} M\right) ; r\right] & =0, \quad r<a, \\
\mathcal{H}_{0}\left[\xi^{-1}\left(b_{2} L+b_{1} M\right) ; r\right] & =2 \mu_{1} d, \quad r<a, \\
\mathcal{H}_{1}[L ; r] & =0, \quad r>a, \\
\mathcal{H}_{0}[M ; r] & =0, \quad r>a .
\end{aligned}
$$

where $b_{1}, b_{2}$ are combinations of elastic constants. We now use Abel transforms to change the Hankel transforms to Fourier transforms; using the inverses of (9) and (10) and similar results for $\mathcal{H}_{1}$, we find

$$
\begin{aligned}
\mathcal{F}_{s}\left\{b_{1} L+b_{2} M ; x\right\}=0, & 0 \leq x<a, \\
\mathcal{F}_{c}\left\{b_{2} L+b_{1} M ; x\right\}=c, & 0 \leq x<a, \\
\mathcal{F}_{s}\{L ; x\}=0=\mathcal{F}_{c}\{M ; x\} a, & x>a .
\end{aligned}
$$

We introduce $\mathcal{F}_{s}\{L ; x\}=\theta(x), \mathcal{F}_{c}\{M ; x\}=\chi(x), 0 \leq x<a$ and extend $\theta(x)$ as odd, and $\chi(x)$ as even, to $-a<x \leq 0$. Then, as in (7), (8),

$$
\mathcal{F}_{c}\{L ; x\}=h[\theta(t) ; x], \mathcal{F}_{s}\{M ; x\}=-h[\chi(t) ; x]
$$

where $h$ is the Hilbert transform. Now equations (11) and (12) yield

$$
b_{1} \theta(x)-b_{2} h[\chi(t) ; x]=0, \quad b_{2} h[\theta(t) ; x]+b_{1} \chi(x)=c, \quad|x|<a .
$$

Put $\chi(x)+i \theta(x)=f(x)$ and combine the equations:

$$
b_{1} f(x)+\frac{b_{2}}{i \pi} \int_{-a}^{a} \frac{f(t) d t}{t-x}=c \quad|x|<a .
$$

Now, following Cauchy, as in (4), and using the Plemelj formulae (5), (6) we obtain the Riemann Hilbert problem

$$
k F^{+}(x)-f^{-}(x)=c^{\prime}, \quad|x|<a ; \quad F^{+}(x)-F^{-}(x)=0, \quad|x|>a
$$

where $k=\left(b_{1}+b_{2}\right) /\left(b_{1}-b_{2}\right)$, and $c^{\prime}$ is a multiple of $c$. Muskhelishvili's technique gives

$$
F(z)=c^{\prime \prime}\{1-X(z)\}
$$


where $X(z)=(z-a)^{i \alpha}(z+a)^{-i \alpha}, \exp (\pi \alpha)=k$. Now the Plemelj formulae yield

$$
f(x)=F^{+}(x)-F^{-}(x)=c^{\prime \prime}\{(a-x) /(a+x)\}^{i \alpha} \quad|x|<a
$$

and the real and imaginary parts of $f(x)$ yield $\theta(x)$ and $\chi(x)$. Finally we find the force/displacement relation $P /\left(2 \mu_{1} h\right)=4 \pi \alpha / b_{2}$.

\section{Conclusions}

Never imagine that you can claim sole authorship of a paper; most of our analysis is obtained by combining the work of others.

\section{References}

1 Morris Kline, Mathematical Thought from Ancient to Modern Times, Oxford University Press, New York, 1972.

2 J. Plemelj, Ein Erganzungssatz zur Cauchyschen Integraldarstellung analytischer Funktionen, Randwerte betreffend, Mon. f. Math. u. Phys. 19 (1908) 205-210.

3 N.I. Muskhelishvili, Some Basic Problems of the Mathematical Theory of Elasticity, Noordhoff, Groningen, 1953.

4 I.N. Sneddon, Mixed Boundary Value Problems in Potential Theory, North Holland, Amsterdam, 1966.

5 G.M.L. Gladwell, Contact Problems in the Classical Theory of Elasticity, Sijthoff and Noordhoff, 1980.

6 I.N. Sneddon, Fourier Transforms, McGraw-Hill, New York, 1951.

7 G.M.L. Gladwell, On inclusions at a bi-material elastic interface, $J$. Elast. 54 (1999) 27-41. 